\title{
Kernos
}

Revue internationale et pluridisciplinaire de religion grecque antique

18 | 2005

Varia

\section{Plutarque et Damon de Chéronée}

Une histoire, un mythe, un texte, ou autre chose encore?

\section{Pierre Ellinger}

\section{(2) OpenEdition \\ 9 Journals}

\section{Édition électronique}

URL : http://journals.openedition.org/kernos/1539

DOI : 10.4000/kernos. 1539

ISSN : 2034-7871

Éditeur

Centre international d'étude de la religion grecque antique

\section{Édition imprimée}

Date de publication : 1 janvier 2005

Pagination : 291-310

ISSN : 0776-3824

\section{Référence électronique}

Pierre Ellinger, «Plutarque et Damon de Chéronée », Kernos [En ligne], 18 | 2005, mis en ligne le 08 juillet 2011, consulté le 01 mai 2019. URL : http://journals.openedition.org/kernos/1539; DOI : 10.4000/kernos.1539 


\title{
Plutarque et Damon de Chéronée : une histoire, un mythe, un texte, ou autre chose encore?
}

\begin{abstract}
Résumé : L'étrange histoire du jeune Damon de Chéronée, le «dernier des Péripoltides », et de son masque de suie, que Plutarque a placée en introduction à ses Vies de Cimon et de Lucullus, a suscité des interprétations fort diverses: dernier avatar du "Chasseur noir», témoignage sur les luttes féroces entre factions pro-romaines et pro-pontiques aux temps de la première guerre de Mithridate... On cherche ici surtout à montrer ce que Plutarque a voulu faire en écrivant cette biographie miniature en écho à celle de Lucullus, le sauveur de sa patrie. Entre des figures de fondateur ou de refondateur qui se substituent les unes aux autres, raconter le passage et l'intégration d'une cité grecque dans l'empire romain, faire un travail de mémoire qui conjure les fantômes du passé et contribue à l'homonoia dans la petite cité. Avec une question centrale : Rome cité-tyran?
\end{abstract}

Abstract: Plutarch and Damon of Chaironeia: a story, a myth, a text, or something more? The strange story of young Damon of Chaironeia, the 'last of the Peripoltids', with his soot-smeared face, which Plutarch placed as an introduction to his Lives of Cimon and Lucullus, has given rise to various interpretations: from a last avatar of the Black Hunter to evidence for the fierce struggle between pro-Roman and pro-Pontic factions at the time of the First Mithridatic War... In this paper we have tried to show what Plutarch's intention may have been when he wrote this miniature biography, echoing that of Lucullus, the saviour of his home city. Between figures of founders or re-founders substituting for each other, the task was to tell of the passing and integration of a Greek city into the Roman Empire, to make a work of memory, liable to ward off ghosts from the past and to contribute to bomonoia in the little city, with a central question : was Rome a tyrant-city?

Il y a un peu plus de dix ans, un jeune chercheur, qui, depuis, nous a donné d'autres travaux remarquables, publiait, dans les Proceedings of the Cambridge Philological Society, un article fort intéressant et amusant à la fois. Article double, dont la première partie était écrite en français et la deuxième en anglais. Intitulé "Black Hunter Variations », Variations sur le Chasseur noir, il portait sur une histoire qui se serait déroulée au $\mathrm{I}^{\text {er }}$ siècle avant notre ère dans la patrie de Plutarque, Chéronée, et que celui-ci raconte en introduction à ses Vies de Cimon et de Lucullus. Un jeune homme du pays, en butte aux avances de l'officier commandant la garnison romaine, et alors que ce dernier, repoussé, se préparait à employer la force, le tuait avec l'aide de quinze de ses compagnons d'âge, puis prenait le maquis. Condamné à mort par la cité, il égorgeait à leur tour les magistrats pendant leur repas en

${ }^{1}$ J. MA, «Black Hunter Variations: I Damon le chasseur noir (Plutarque, Cimon, 1-2); II Damon of Chaironeia: a Historical Commentary (Plut. Kim. 1-2); III Damon of Chaironeia: Battlelines », PCPhS 40 (1994), p. 49-80. Il suffit de citer, récemment traduit en français, du même auteur, Antiochos III et les cités de l'Asie mineure occidentale, Paris, les Belles Lettres, 2003. 
commun, puis ravageait par ses raids le territoire, jusqu'à ce que la cité, par force négociations et ambassades, l'apaise, l'induise à revenir, l'élise même gymnasiarque, et l'assassine, dans l'étuve du gymnase, alors qu'il s'enduisait d'huile. La première partie de l'article était une analyse structurale de l'épisode et reconnaissait dans le jeune homme qui se barbouillait, lui et ses compagnons, le visage de suie, pour assaillir, au petit matin, à l'heure entre chien et loup, l'officier romain au sacrifice, avant de s'enfuir dans les eschatiai, un dernier avatar du Chasseur noir cher à Pierre Vidal-Naquet. Cette partie était écrite en français, car, dit-on, seule cette langue permet les effets de style nécessaires au succès de ce type d'analyse. La deuxième partie, en anglais, bien sûr, représentait la méthode opposée, et était un commentaire historique, positif, quasi ligne à ligne, du même épisode, d'une érudition époustouflante. Le double «à la manière de » était, dans sa duplicité, digne du Chasseur noir, rusé et même perfide, frisant le canular. Le nombre des seize compagnons béotiens renvoie aux seize ans des jeunes éphèbes des Apatouries... athéniennes, la montagne structuraliste accouche d'une souris historiciste: le Chasseur noir s'est enfin incarné dans la réalité ! Le commentaire historique fait mine de s'interroger le plus sérieusement du monde sur les possibilités de récession des industries de roseaux pour flûte et de parfums pour oublier les maux des cités du lac Copaïs, induites par les événements ${ }^{2}$. Enfin une troisième partie, mais évidemment elle-même double, revenait de manière critique sur les deux analyses précédentes. Dénonçant le dialogue de sourds des deux méthodes, elle se voulait une protestation contre la situation faite aux jeunes chercheurs, sommés de prendre parti dans la moderne stasis entre les deux factions. Le bilan, toutefois, me paraît marquer une certaine déception. L'énergie épuisée à inventer la topogaphie de cette nouvelle bataille de Chéronée, le dialogue espéré qui surmonterait les oppositions ne prend pas. Après que l'amusement de la méta-recherche s'est évanoui, «the fun of metascholarship has died away " (l'anglais va nettement mieux cette fois que le français), que me reste-t-il, s'interroge tristement le jeune chercheur déguisé en Chasseur noir. Qu'a-t-il retiré de ce double essai peu tempéré des deux méthodes? La dernière phrase, en forme de conseil, fait allusion au vin pur qu'auraient bu Damon et ses compagnons avant leur coup, pour se donner du courage, ou signer symboliquement leur passage à la sauvagerie, selon l'interprétation retenue. Si je ne le surinterprète pas à mon tour, le conseil ne s'applique pas qu'aux autres: "Don't drink your wine neat». Ne buvez pas votre vin pur! Une fin bien dans le ton. Le surlendemain des Apatouries, dont devaient se souvenir, non sans nostalgie, les citoyens athéniens, comme du jour de leur première entrée dans l'âge adulte, ne s'appelait-il pas Epibda, c'est-à-dire, en français, la "gueule de bois ", hangover en anglais ${ }^{3}$ ?

Comme on pouvait un peu s'y attendre, cet article de John Ma est passé dans notre ciel scientifique comme un météore isolé. Bien qu'accueilli

\footnotetext{
2 MA, l.c. (n. 2), p. 50, 57, 65.

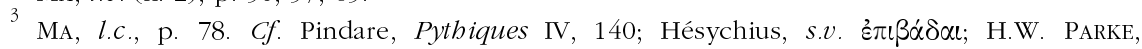
Festivals of the Athenians, Londres, 1977, p. 92.
} 
élogieusement à la fois par Fergus Millar et Pierre Vidal-Naquet ${ }^{4}$, il a plutôt suscité, me semble-t-il, une prudente réserve. Pourtant, l'attention attirée sur l'épisode plutarchéen, les études se sont multipliées ${ }^{5}$. Mais seule la dernière en date, de Carlo Franco, en 2003, rend quelque peu justice au travail pionnier de J. Ma ${ }^{6}$. Christopher Mackay ne veut pas prendre au pied de la lettre le texte de Plutarque, mais cherche à débusquer les cruelles luttes de factions que la tradition locale aurait choisi d'occulter, entre pro-Romains et pro-Pontiques. Leur alternance au pouvoir expliquerait les incohérences apparentes de la politique chéronéenne. L'épisode est restitué dans une chronologie très courte, entre l'hiver 88/87 et l'hiver $87 / 86$, avant la grande bataille où, au printemps 86, Sylla affronte à Chéronée les troupes d'Archélaos et de Taxile $^{7}$. Au contraire, pour John Thornton, les brigandages de Damon auraient duré quelques années au-delà des batailles de Chéronée puis d'Orchomène, après la fin de la première guerre mithridatique. Il propose une troisième grille d'analyse, cette fois en italien, inspirée d'Eric Hobsbawm, avec la figure du brigand expression des aspirations des masses populaires ${ }^{8}$. C. Franco prolonge encore plus l'aventure de Damon, puisque, pour lui, on aurait dû attendre qu'il ait atteint l'âge légal de trente ans pour le nommer gymnasiar$q^{9} e^{9}$ ! Pour la chronologie, on est donc d'accord au moins sur le point de départ de l'affaire, qui est celui par lequel Plutarque la rattache à son propos : le passage de Lucullus par Chéronée "pour une certaine mission », son enquête sur les événements, qui le conduit à retirer la garnison et à l'emmener avec lui. Le seul moment où il aurait eu le pouvoir de prendre une telle décision était quand il avait été envoyé en avant-garde par Sylla - dont il était le questeur - lors de son avance en Grèce au printemps $87^{10}$. La garnison

${ }^{4}$ F. Millar, «Greece and Rome from Mummius Achaicus to St Paul: Reflections on a Changing World", in J.-Y. MARC, J.-Ch. MORETTI (éds), Constructions publiques et programmes édilitaires en Grèce entre le II siècle av.J.-C. et le II siècle ap. J.-C., Athènes/Paris, 2001 (BCH, Suppl. 39), p. 8; P. VIDAL-NAQUET, Mémoires 2, Paris, Seuil/La Découverte, 1998, p. 228. Cf. aussi O. Murray, "Pierre Vidal-Naquet et le métier d'historien de la Grèce : l'école de Paris' ", in F. Hartog, et al. (éds), Pierre Vidal-Naquet, un historien dans la cité, Paris, 1998, p. 157.

R.M. Kallet-MarX, Hegemony to Empire. The Development of the Roman Imperium in the East from 148 to 62 B.C., Berkeley/Los Angeles, 1995, p. 279-282; C.S. MACKAY, "Damon of Chaeronea: the Loyalties of a Boiotian Town during the First Mithridatic War », Klio 82 (2000), p. 91-106; J. Thornton, Lo storico, il grammatico, il bandito. Momenti della resistenza greca all'Imperium Romanum, Catane, 20012, p. 215-247; C. Franco, « Anni difficili. Plutarco e Damone di Cheronea (Cim. 1-2.2)», in B. VIRGILIO (éd.), Studi ellenistici XV, Pise, 2003, p. 191-213. Antérieurement, voir surtout M. HollEAuX, «Décret de Chéronée relatif à la première guerre de Mithridates », REG 32 (1919), p. 320-337 = Études d'épigraphie et d'bistoire grecques I, Paris, 1938, p. 143-159; A. Blamire, Plutarch. Life of Kimon, Londres, 1989 (BICS Suppl. 56), p. 79-84.

6 FRANCO, l.c. (n. 5), p. 192

MaCKay, l.c. (n. 5), en particulier p. 93-94, 98-105.

Thornton, o.c. (n. 5), p. 226-227, 245, pour la durée de l'aventure de Damon; 233-247, pour la figure du bandit.

9 Franco, l.c. (n. 5), p. 208-209.

10 Plutarque, Cimon, 1, 6 et Sylla, 11, 8; déjà Holleaux, o.c. (n. 5), I, p. 153-154. Pour la date, discussion détaillée dans A. KeAveney, Lucullus. A Life, Londres/New York, 1992, p. 18-19 et 212 n. 11; MACKAY, l.c. (n. 5), p. 93 n. 4 et 100. 
et l'officier coupable, probablement une cohorte et son tribun militaire ${ }^{11}$, appartiendraient alors aux troupes du légat Q. Braetius Sura, dépêché en 88 par le proconsul de Macédoine C. Sentius, pour lutter contre les troupes d'Archélaos, le général de Mithridate, et qui avaient déjà affronté par trois fois celles-ci avec succès, autour de Chéronée précisément ${ }^{12}$. C'est pour la durée de la suite que l'on diverge : quelques mois, quelques années ou plus d'une dizaine; personnellement, la solution intermédiaire me paraîtrait plus plausible. Le texte de Plutarque, ce qui nous ramène au titre de cette étude, a été considéré, alternativement ou simultanément, comme une tradition orale authentique de Chéronée ${ }^{13}$, comme un document à exploiter pour éclairer un pan d'histoire peu connu ${ }^{14}$, ou, mais encore insuffisamment à mon sens, comme un texte de Plutarque, à étudier d'abord en tant que texte ${ }^{15}$. Pour ce qui me concerne, je me suis depuis longtemps intéressé à lui d'un double point de vue, qui tient à mes préoccupations phocidiennes ${ }^{16}$ : du point de vue d'abord de l'étude des récits, puisque le déguisement de Damon et de ses compagnons attaquant l'espace sacrificiel dans la lumière incertaine de l'aube, le visage masqué de suie, était souvent invoqué en parallèle pour les Choisis phocidiens entièrement blanchis de gypse, attaquant le camp thessalien, à la lueur de la pleine lune, dans leurs guerres d'indépendance de la fin de l'époque archä̈que ${ }^{17}$. Et ensuite, en quelque sorte en voisin. Chéronée se trouve à $13 \mathrm{~km}$ à vol d'oiseau d'Hyampolis. Plutarque y venait - c'est la scène d'un de ses Propos de table ${ }^{18}$ - assister aux Elaphébolia au sanctuaire d'Artémis, localisé aujourd'hui à Kalapodi, pour les commémorations de la grande victoire du Désespoir phocidien, remportée par Daïphantos d'Hyampolis, le libérateur de la Phocide, par ailleurs un de ses ancêtres ${ }^{19}$. L'histoire de

11 MackaY, l.c. (n. 5), p. 92 n. 2. Très exactement, cette troupe hiverne à Chéronée.

12 Plut., Sylla, 11, 7; Appien, Mithridatica, 29, 114.

13 Cf. Plut., Cimon, 1, 8: "à ce que racontent nos pères » (selon la traduction d'A.-M. Ozanam in F. Hartog (éd.), Plutarque, Vies parallèles, Paris, Gallimard, 2001 [Quarto], que j'utiliserai désormais, sauf avis contraire, en la modifiant parfois légèrement); J. BUCKLER, "Plutarch and Autopsy », in ANRW II, 33.6 (1992), p. 4806; MA, l.c. (n. 1), p. 60-61; FrANCO, l.c. (n. 5), p. 194. Le fait de reconnaître là une «tradition orale » a pu conduire aux deux attitudes opposées, le refus total d'un "récit populaire » forgé de toutes pièces pour expliquer l'épithète d'asbolômenoi donnée à certains habitants de la région (J. van OOTEGHEM, L. Licinius Lucullus, Bruxelles, 1959, p. 37 n. 3), ou, au contraire, l'acceptation de "l'authenticité des faits », après croisement avec d'autres documents (ainsi R. Flacelière, notice de la Vie de Cimon, in Plutarque. Vies, t. VII, Paris, Les Belles Lettres, 1972 [CUF], p. 2-3).

14 MACKAY, l.c. (n. 5), p. 91.

15 FrANCO, l.c. (n. 5), p. 193-197, 212-213.

16 P. Ellinger, La Légende nationale phocidienne. Artémis, les situations extrêmes et les récits de guerre d'anéantissement, Athènes/Paris, 1993 (BCH, Suppl. 27).

17 Hérodote, VIII, 27; Pausanias, X, 1, 11; le premier rapprochement, à ma connaisance, déjà dans C.A. LOBECK, Aglaophamus sive de Theologiae mysticae Graecorum causis, Koenigsberg, 1829, I, p. 654-655.

Propos de table IV, 660d.

19 Plutarque, Des délais de la justice divine, 558a-b. Plutarque raconte la victoire phocidiennne du Désespoir dans ses Vertus des femmes, 244b-e. Il avait écrit une Vie, perdue, de Daïphantos, dont il égale les exploits à ceux de Miltiade à Marathon, ou d'Épaminondas et 
Damon, elle-même, se termine pour partie en Phocide, à Stiris, plus connue aujourd'hui pour le monastère d'Hosios Loukas ${ }^{20}$. Ses descendants s'y étaient réfugiés, constituant un genos qui portait encore avec fierté, du temps de Plutarque, le nom d'asbolômenoi, les «Couverts de suie », en souvenir de leur ancêtre $^{21}$. Ces étroites préoccupations locales me faisaient, pour ma part, apprécier tout autant la partie la plus classique que la partie structurale de l'étude de J. Ma. Mais si, à mes yeux, son intuition, qui n'est pas, et de loin, uniquement ironique - il ne faut pas s'y tromper - de voir en Damon un lointain «descendant » du Chasseur noir, est toujours justifiée, il est, je crois, possible aujourd'hui d'aller un peu plus loin. En montrant qu'à l'évidence son surnom de Péripoltas apparente Damon aux péripoloi, son masque de suie qui le cache aux cryptes et qu'il est un éphèbe qui échoue, J. Ma signalait aussi que les catégories qui sous-tendent ces institutions, tout autant que le nouveau récit ici produit, fonctionnaient encore à une époque jugée aussi tardive $^{22}$. En d'autres termes, pas plus que la religion grecque et la cité, le «mythe » n'est mort, c'est le cas de le dire, à Chéronée. Il faut alors en tirer toutes les conséquences et essayer de voir aussi comment il s'ancre dans les réalités de ce temps.

Plutarque, presque immédiatement, commence par une notation essentielle. Ce Damon, dernier descendant d'une noble et prestigieuse lignée, était un orphelin. Il descendait du devin Péripoltas, dont il tirait son surnom, qui avait, aux côtés du roi Opheltas, conduit, soixante ans après la guerre de Troie, la migration des Béotiens, depuis la Thessalie, jusque dans leur nouvelle patrie ${ }^{23}$. Nous savons malheureusement peu de choses sur ces traditions, mais les bribes qui en ont survécu méritent peut-être d'être signalées pour notre propos, puisque l'on y rencontre déjà une histoire de jeunes, de vin, de blanc et de noir. Un oracle avait prédit aux Béotiens qu'ils seraient chassés de

Pélopidas à Leuctres (De l'impossibilité de vivre heureux en suivant Épicure, 1099e-f). Cf. ELLINGER, o.c. (n. 16), p. 15 et 235-236.

${ }^{20}$ Cf. J. MCINERney, The Folds of Parnassus. Land and Ethnicity in Ancient Phokis, Austin, 1999 , p. 319-320.

${ }_{22}$ Cimon, 1, 9.

22 MA, l.c. (n. 1), p. 49-52, 56, 73-74. Si l'on cherche un autre « Chasseur noir » pour la même époque, on le trouvera aisément dans les traditions sur l'enfance de ... Mithridate lui-même, rapportées par Justin, XXXVII, 2, 9 (et XXXVIII, 8, 1) : "Craignant ensuite qu'à défaut de poison, ses ennemis n'employassent le fer, il feignit une vive passion pour la chasse. Ainsi pendant sept années, ni à la campagne, ni à la ville, il ne coucha sous l'abri d'un toit, mais, errant au fond des bois, il passait la nuit sur différents points des montagnes, sans que personne connût sa retraite. Là, il s'habituait tantôt à fuir, tantôt à poursuivre les bêtes sauvages, quelquefois même il éprouvait ses forces contre elles. Par ce genre de vie, il évita les embûches, et endurcit son corps à toutes les souffrances » (trad. J. Pierrot, E. Boitard). Texte à lire lui aussi en clef grecque et pas seulement d'enfance royale iranienne. Étonnant commentaire-paraphrase de Th. REINACH, Mithridate Eupator, roi de Pont, Paris, 1890, p. 53-54; voir également G. Windengren, « La légende royale de l'Iran antique », Hommages à Georges Dumézil, Bruxelles, 1960 (Coll. Latomus, 45), p. 227-230; B.C. MCGIng, The Foreign Policy of Mithridatus VI Eupator, King of Pontus, Leyde, 1986 (Mnemosyne, Suppl. 89), p. 43-44.

${ }^{23}$ Cimon, 1, 1; cf. Thucydide, I, 12, 3. 
leur pays, Arné de Thessalie, un pays de la terre blanche, signe de richesse et d'opulence $^{24}$, le jour où les corbeaux deviendraient blancs. Des jeunes gens, ayant trop bu, eurent l'idée saugrenue de blanchir des corbeaux avec du gypse et de les relâcher. L'oracle s'était réalisé ${ }^{25}$. Ceux des Béotiens qui étaient trop attachés à leur terre préférèrent entrer dans un contrat de servitude et devenir les pénestes des conquérants thessaliens ${ }^{26}$, mais les plus courageux, qui choisirent la liberté plutôt que l'esclavage, partirent. Leur errance, sous la double direction du roi Opheltas et du devin Péripoltas (on peut se demander si effectivement le nom de ce dernier n'y fait pas non plus allusion ${ }^{27}$ ) les conduisit finalement dans le pays des Minyens et des Cadméens. Le lieu par lequel ils y entrèrent fut évidemment Chéronée, qui fut la première cité de la nouvelle Béotie fondée par eux ${ }^{28}$, sous le nom à nouveau d'Arné, la nouvelle Arné $^{29}$.

Péripoltas et ses descendants, nous dit Plutarque, se fixèrent à Chéronée. C'était une race de guerriers courageux. Beaucoup périrent en défendant le pays contre les Barbares, lors de l'invasion de Xerxès, puis de celle des Galates $^{30}$. Remarquons les jalons, peut-être un peu étranges pour nous, de cette temporalité, qui conduisent du temps des origines à celui de Plutarque : la deuxième guerre médique et directement en pleine époque hellénistique, aux débuts de la domination étolienne sur la Grèce centrale. L'époque classique est sautée d'un coup, avec toute la guerre du Péloponnèse, Leuctres et la bataille contre Philippe ${ }^{31}$. Le lion surmontant le tombeau commun du Bataillon sacré thébain était tout de même le monument le plus remarquable de Chéronée ${ }^{32}$ ! En tout cas, de ces braves, il n'en restait plus qu'un, notre héros,

\footnotetext{
${ }^{24}$ Strabon, IX, 5, 18. La région est dominée par les hauteurs du blanc Titane, dont le nom évoque les mêmes associations. Sur les valeurs d'âge d'or et de pays de cocagne de la terre blanche, $c f$. ElLINGER, o.c. (n. 16), p. 95-104.

25 L'oracle des corbeaux est raconté dans sa version la plus complète par l'Atthidographe Démon, FGrHist $327 \mathrm{~F} 7$ et dans les proverbes de Zénobius (III, 87 éd. E.L. LeuTsch et F.G. SchneIDEwIn, Paroemiographi Graeci, Göttingen, 1839, I, p. 78-79). Sources le plus commodément rassemblées par Jacoby dans son commentaire à Démon (vol. III b [Suppl.], p. 209-210) et discutées par G. HuXLEY, « White Ravens », GRBS 8 (1967), p. 199-202.

26 Cf. Archémachos d'Eubée, FGrHist 424 F 1 = Athénée, VI, 264a-b; Pausanias l'Atticiste, Fragmenta, $\pi$ 16, p. 204 (éd. H. ERBSE, Untersuchungen zu den attizistischen Lexika, Berlin, 1950 [Abhandl. Deutschen Akad. Wiss. Berlin. Philosophisch-historische Kl. 1949, 2]); textes n ${ }^{\circ} 2$ et 20 dans J. Ducat, Les Pénestes de Thessalie, Paris, 1994, p. 14-16, 38-40, avec commentaire.

${ }_{27}$ MA, l.c. (n. 1), p.60; MACKAY, l.c. (n. 5), p. 98-99.

28 Cimon, 1, 1 : "Chéronée, la première cité qu'ils occupèrent, après en avoir chassé les Barbares ». Ces Barbares étant, selon Strabon (IX, 2, 3), les Pélasges qui se réfugièrent en Attique, les Thraces refoulés sur le Parnasse, et les Hyantes qui fondèrent Hyampolis en Phocide.

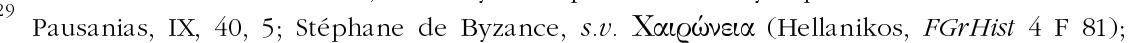
scholie à Thucydide, I, 12, 3, p. 16 éd. HudE.

Cimon, 1, 1.

31 On la retrouve en revanche dans la conclusion de la Vie de Cimon, 19, 3-4.

32 Voir la description de la cité par Pausanias, IX, 40, 10. Plutarque et la défaite de Chéronée en 338 : Camille, 17, 8 (jour sinistre pour les Grecs); Alexandre, 9, 2 et surtout Démosthène, 1920 .
} 
Damon, le dernier des Péripoltides, pourrait-on dire. Or c'était un orphelin. Cette rupture dans la transmission avait ses conséquences désastreuses inéluctables; s'il était beau de corps et d'une âme fière, surpassant tous ses compagnons d'âge, il était en revanche sans éducation et rude de caractère

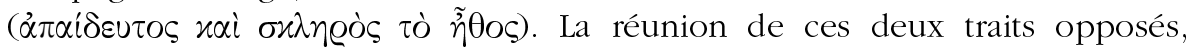
positif et négatif, est, me semble-t-il, une marque essentielle de tout le récit, comme une figure de rhétorique, qui en est la matrice narrative, la signature distinctive qui va se propager de bout en bout et qu'il faut bien se garder, à

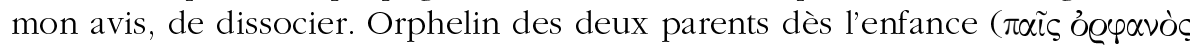

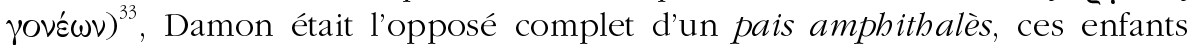
que l'on plaçait en tête des grandes processions civiques, en particulier en Béotie. Choisis justement, comme le signifie leur nom, parce qu'ils avaient leurs deux parents en vie, ils étaient les représentants symboliques de l'avenir de la cité, présage heureux de prospérité future ${ }^{34}$. Autrement dit, cet enfant était la parfaite expression de ce que Plutarque précise ensuite : « Notre patrie était dans une triste situation et se trouvait méprisée à cause de sa petitesse et de sa pauvreté $\|^{35}$.

L'affaire homosexuelle a été abondamment commentée, du point de vue romain aussi bien que du point de vue grec, et de la relation entre les deux cultures $^{36}$. Du point de vue romain, en évoquant l'histoire du miles Maria$n u s^{37}$, ce soldat qui tue son supérieur qui cherchait à profiter de sa position d'autorité, et qui est acquitté et même félicité par Marius, leur général. J. Ma, au contraire, suggère que notre Romain, probablement de bonne famille, était philhellène et cultivé, ayant peut-être même lu son Platon sur les mœurs béotiennes. Il courtise, au moins en un premier temps, son aimé à la grecque, par la persuasion et en lui offrant des cadeaux. La réaction de Damon n'étaitelle pas excessive, due à son manque d'éducation et son caractère rude, sinon au sang chaud, bien connu, des Béotiens ${ }^{38}$ ? En d'autres temps, en d'autres

33 Cimon, 1, 2. Il n'était donc pas, à la différence d'un autre personnage de Plutarque, qui lui ressemble par certains côtés, Coriolan, flanqué d'une redoutable mère; $c f$. Coriolan, 1, 2-5, et, pour le rapprochement, THORNTON, o.c. (n. 5), p. 220 n. 18.

34 Cf. A. OEPKE, "A 'A $\varphi \imath \theta \alpha \lambda \varepsilon i \varsigma$ im griechischen und hellenistischen Kult », $A R W 31$ (1934), p. 42-56; L. ROBERT, «A $A \varphi \varphi \imath \alpha \lambda \hat{n} \varsigma$ », HSPh, Special Volume W.S. Ferguson (1940), p. $509-519$ (= OMS I [1969], p. 633-643). Cet enfant est littéralement un « rejeton florissant des deux côtés ». À Thèbes, un pais amphithalès marchait en tête de la procession des Daphnéphories, elle-même reliée à l'antique conquête de la Béotie (Proclus, Chrestomathie, 70 et 76, avec le commentaire d'A. SEveryns, Recherches sur la Chrestomathie de Proclos I, II, Paris, 1938, p. 214-229). Les amphithaleis portaient l'eirésionè, figuraient dans les processions de mariage, coupaient les couronnes pour les vainqueurs aux jeux.

35 Cimon, 1, 3 (dans la traduction de R. Flacelière)

36 Cf. Ma, l.c. (n. 1), p. 61-62; MACKAY, l.c. (n. 5), p. 93-94; THORNTON, o.c. (n. 5), p. 240-241; et surtout Franco, l.c. (n. 5), p. 198, 201-203.

37 Plutarque, Marius, 14, 4-9; Apophtegmes de rois et de généraux, 202b; Cicéron, Pro Milone, 9; Valère-Maxime, VI, 1, 12; [Quintilien], Declam. maj., III; C.A. WILlIAMS, Roman Homosexuality. Ideologies of Masculinity in Classical Antiquity, Oxford/New York, 1999, p. 110-111.

MA, l.c. (n. 1), p. 61-62. 
lieux, les choses auraient pu se passer autrement ${ }^{39}$. De son côté, le Romain aurait pu ne pas jouer l'occupant brutal, et voyant le jeune homme réticent, le laisser libre. Mais il n'est ni l'Agésilas de Xénophon et de Plutarque, renonçant à son désir pour un jeune Perse, fils d'un de ses alliés ${ }^{40}$, ni Scipion l'Africain, après la prise de Carthagène, libérant la jeune captive espagnole offerte en cadeau par la troupe et la renvoyant à son père ${ }^{41}$. Ce que je voudrais montrer, c'est que l'affaire n'est pas simple ni susceptible d'une solution univoque, telle que le seul sens de l'honneur, l'indignation devant le sort fait à un homme libre, traité comme un esclave. On s'est aussi interrogé sur la portée politique de l'incident: pure affaire privée, prétexte cachant la vraie conspiration politique des partisans de Mithridate pour C.S. Mackay, ou porteuse de connotations antityranniques pour $\mathrm{C}$. Franco ${ }^{42}$. Cette dernière intuition se rapproche du centre de la cible. Assez curieusement, aucun des commentateurs n'a exploité le parallèle le plus évident, et qui ne pouvait manquer de venir à l'esprit des compatriotes de Plutarque et surtout de lui-même quand il entamait la série des Vies parallèles, en donnant la place d'honneur à des thèmes qui évoquaient sa Béotie natale : avec les Vies d'Épaminondas, la première de toutes, de Pélopidas, puis celles de Lucullus et de Sylla, les deux Romains liés à Chéronée ${ }^{43}$. Il ne s'agit pas moins de ce que l'on peut appeler le mythe national béotien, autrement dit les traditions autour de la bataille de Leuctres! Peut-être les commentateurs ont-ils été trompés - je dirais presque qu'ils sont tombés dans le piège de Plutarque - par l'ouverture même du récit qui, on l'a vu, ne fait curieusement allusion, comme événements marquants du passé, qu'à l'invasion de Xerxès et à celle des Galates. Pourtant Plutarque avait raconté la célèbre histoire des jeunes filles de Leuctres au moins dans son Pélopidas. Xénophon y faisait déjà allusion et on la retrouve, parmi une multitude de sources, chez Diodore, Pausanias, de même que dans les Histoires d'amour de la collection des Moralia, qui donnent la version la plus détaillée, que je suivrai en priorité ${ }^{-44}$. Deux jeunes Spartiates, aux noms tout aussi évocateurs chez Pausanias - Phrourarchidas et Parthénios ${ }^{45}$ - que le

39 Pour ne faire allusion qu'aux coutumes crétoises (Strabon, X, 4, 21).

40 Plutarque, Agésilas, 11, brodant sur Xénophon, Agésilas, 4, 5.

Cf. Polybe, X, 19, 3-7; Tite-Live, XXVI, 50, 1; rapprochement judicieux par ThORNTON, o.c. (n. 5), p. 240-241. Une autre version moins édifiante dans Aulu-Gelle, VII, 8, 3-6.

MACKAY, l.c. (n. 5), p. 93; Franco, l.c. (n. 5), p. 212-213; déjà THORNTON, o.c. (n. 5), p. 241 n. 77 citant Polybe sur les exactions sexuelles typiques de la dégénérescence d'une royauté ou d'une aristocratie en tyrannie (VI, 7, 7-9 et VI, 8, 5).

43 Cf. F. Frazier, in Hartog, o.c. (n. 13), p. 1987; C. Pelling, Plutarch and History, Londres, 2002 , p. 31 n. 14.

Xénophon, Helléniques VI, 4, 7; Diodore, XV, 54, 1-3; Plutarque, Pélopidas, 20-22; De la malignité d'Hérodote, 856f; Pausanias, IX, 13, 5-6. Pour les Histoires d'amour, 3, 773b-774d, faussement attribuées à Plutarque, mais bien renseignées sur les traditions locales béotiennes, $c f$. M. Cuvigny, Plutarque. Euvres morales, t. X, Paris, Les Belles Lettres, 1980 (CUF), p. 111-117, 127-130, 157-159, avec liste complète des autres sources; voir également A. SCHACHTER, Cults of Boiotia 2, Herakles to Poseidon, Londres, 1986 (BICS, Suppl. 38, 2), p. 122; J. FOnTENROSE, The Delphic Oracle, Berkeley/Los Angeles/Londres, 1978, p. 145-148.

45 Pausanias, IX, 13, 5. 
surnom de Damon, Péripoltas, allaient consulter à Delphes. Étant donné les règles spartiates réservant une telle consultation aux rois, ce ne pouvaient être que des Pythiens envoyés par eux, et, de fait, Diodore les qualifie d'« ambassadeurs des Lacédémoniens ${ }^{46}$, donc des représentants on ne peut plus officiels de la cité. Au retour de leur mission, ils demandèrent, comme à l'aller, l'hospitalité chez Skédasos, un paysan du village de Leuctres: ses filles leur avaient particulièrement plu. Le père était absent, les filles les accueillirent selon l'usage de la maison; ils abusèrent d'elles, puis devant la véhémence de leurs protestations, les tuèrent, et les jetèrent dans un puits ${ }^{47}$. Le père, de retour, découvre les cadavres, enquête auprès des voisins, comprend ce qui s'est passé $e^{48}$. Jusque-là, la similitude avec l'histoire de Damon n'est sans doute pas évidente, mais voici la suite. Skédasos partit pour Lacédémone demander justice. Or sur la route, il rencontra un autre vieillard qui en revenait. L’homme était d'Oréos en Eubée. Et là, l'harmoste lacédémonien ${ }^{49}$, s'étant épris de son fils et ne réussissant pas à le persuader, tenta d'employer la force et de l'enlever à la palestre. Mais le pédotribe et les autres jeunes (neaniskoi) l'en empêchèrent. Sur le moment, il n'insista pas, mais le lendemain, il arma une trière, enleva le garçon (meirakion), passa sur le rivage opposé, sur le continent, et tenta de lui faire violence; devant sa résistance, il l'égorgea

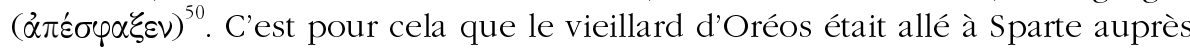
des éphores, qui l'avaient éconduit. Inutile donc, disait-il à Skédasos, de s'y rendre. Mieux valait rentrer donner un tombeau à ses filles. Mais celui-ci s'était obstiné. Or ni les éphores ne voulurent l'entendre, ni ensuite aucun des deux rois, ni même un seul des simples citoyens. Skédasos court à travers Sparte, prend le soleil à témoin, et, invoquant les Érinyes, se donne la mortt ${ }^{51}$. L’histoire du vieillard d'Oréos ne figure pas dans la Vie de Pélopidas, mais le thème du refus de justice à Sparte y est bien présent. Dans cette version, Skédasos rentre à Leuctres, et, après avoir maudit Sparte, s'égorge sur le tombeau de ses filles ${ }^{52}$. La suite est connue, c'est auprès de ce tombeau que se déroulera la bataille de Leuctres, accomplissant le mènima des Leuctrides ${ }^{53}$.

46 Diodore, XV, 54, 3

[Plut.], Histoires d'amour, 3, 773b-d. Dans les autres versions, elles se tuent, de diverses manières, pendaison, poignard

48 Histoires d'amour, 3, 773d-e.

49 Il se nomme Aristodème dans le récit. Au contraire, l'officier romain reste anonyme.

Ibid., 773e-f, à comparer, pour les termes identiques employés, avec Cimon, 1, 3. Le meurtrier fait ensuite bombance.

51 Histoires d'amour, 3, 774a-b.

52 Pélopidas, 20, 6; de même pour Pausanias, IX, 13, 5. Chez Diodore, ce sont les filles ellesmêmes qui avaient maudit les Spartiates.

53 Mènima : Plut., Pélopidas, 20, 7; Pausanias, IX, 13, 5; cf. [Plut.], Histoires d'amour, 3, 774d (prophétie donnée en songe par Skédasos à Pélopidas). On a fait remarquer que le nom de Skédasos pourrait être signifiant dans ce contexte, s'il vient bien du verbe $\sigma \varkappa \varepsilon \delta \dot{\alpha} \nu \nu u \mu$, actif : "verser le sang " (Iliade VII, 330), passif : "se disperser de tous côtés » pour une armée en déroute (Thucydide, IV, 56; VI, 52); employé également déjà par Hésiode pour les maux de la jarre de Pandora (Travaux, 95); cf. PFISTER, s.v. "Skedasos», RE III A (1927), col. 467, 42-55; SCHACHTER, O.C. (n. 44), p. 122 n. 3. 
Cette histoire relève d'un groupe de récits fort étendu et productif, celui des récits sur les viols de jeunes filles. Quand elles sont un grand nombre, un choros entier, le récit peut justifier l'asservissement du peuple agresseur : c'est l'histoire bien connue du viol des jeunes filles spartiates au sanctuaire d'Artémis Limnatis aux frontières de la Messénie et de la Laconie ${ }^{54}$. Quand la jeune fille est seule, c'est généralement une histoire de tyran; et un troisième personnage y intervient souvent, le jeune défenseur ou vengeur de la jeune fille; ces histoires sont souvent aussi associées à Artémis ${ }^{55}$. Avec un motif fréquent, que nous retrouvons dans l'histoire de Damon, celui de la ruse et du déguisement, sous la forme cette fois du travestissement sexuel : le jeune homme se déguise en fille pour s'introduire auprès du tyran ${ }^{56}$. Dans une histoire qui appartient de manière périphérique au même groupe, mais qui est totalement ahistorique, puisque l'affaire se passe dans le monde divin, la proximité est encore plus grande: le dieu Alphée ne pouvant obtenir les faveurs d'Artémis elle-même par la persuasion et les prières, se décide à employer la force et, pour cela, se rend à une fête où Artémis devait danser avec ses compagnes les Nymphes. Mais la déesse, qui a eu vent du projet, s'enduit, elle et ses compagnes, le visage de boue et échappe au viol, ne pouvant être reconnue. On n'est pas très loin de Damon et du choros de ses compagnons d'âge au visage enduit de suie ${ }^{57}$. Mais revenons à la signification politique de cet ensemble. La Vie de Cimon, un peu plus loin, présente une autre histoire de ce type : celle du régent Pausanias de Sparte, au moment où il aurait voulu se faire «le tyran de la Grèce ». Il fait venir pour abuser d'elle une jeune fille noble de Byzance, une de ces cités alliées qu'il est censé mener au combat pour la liberté, et la tue par méprise. L'affaire signe la fin de cette première hégémonie spartiate, pour annoncer le début de l'hégémonie athénienne et la fondation de la Ligue de Délos. Cette histoire, comme je le montre ailleurs, forme charnière et marque l'adaptation de ce type de récits à des circonstances historiques nouvelles ${ }^{58}$. La vraie menace n'est plus celle d'un homme qui voudrait devenir tyran à l'intérieur de la cité, mais d'une cité tyran des autres cités, polis-tyrannos ${ }^{59}$. Or c'est bien cela ce que signifie l'histoire des Leuctrides, comme le confirme, de manière parfaitement limpide, son deuxième volet, dans les Histoires d'amour, l'épisode de l'harmoste d'Oréos.

54 Pausanias, IV, 4, 1-3; cf. Ellinger, o.c. (n. 16), p. 41-43.

55 Cf. Antoninus Liberalis, XIII, 3-7; Pausanias, VIII, 5, 11-12; VIII, 47, 6; saint Jérôme, Adversus Jovinianum I, 308a (Patrologie latine, 23, 272). Dossier étudié dans mon livre, La fin des maux. D'un Pausanias à l'autre (Les Belles Lettres, sous presse).

56 Antoninus Liberalis, XIII, 3-7; Héraclide du Pont, FHG II, 222 fr. 32.

Pausanias, VI, 22, 9 (cf. Ellinger, o.c. [n. 16], p. 40); à comparer avec Cimon, 1, 4 et 8, pour l'étroite similitude des expressions employées.

58 Cimon, 6; également : Sur les délais de la justice divine, 10, 555c; Pausanias, III, 17, 7-9; Aristodèmos, FGrHist 104, 4-9. Pausanias le régent, «tyran de la Grèce »: Hérodote, V, 32. Analyse complète dans La fin des maux (supra, n. 55).

Cf. Thucydide, I, 69, 1; II, 63, 2; III, 37, 2; VI, 85, 1; K.A. RAaflaub, « Polis Tyrannos: Zur Entstehung einer historischen Metapher », in G.W. BOWERSOCK, W. BuRKERT, M.C.J. PutNAM (éds), Arktouros. Hellenic Studies presented to B.M.W. Knox, Berlin/New York, 1979, p. 237-252. 
Donc ce qu'implique le parallélisme évident avec l'histoire de l'officier romain commandant la garnison hivernant à Chéronée, ce n'est pas tant le risque d'une mise en série des Perses, des Galates et des Romains, où Damon reprendrait le flambeau de la lutte de ses ancêtres pour la défense de la patrie contre les Barbares. Personne ne peut vraiment plus comparer, en ce Ir siècle avant J.-C., les Romains à des Barbares, aux Perses peut-être, mais plus difficilement aux Galates ${ }^{60}$. C'est bien plutôt le risque de la mise en série de la domination romaine avec les hégémonies athénienne, lacédémonienne, et vraisemblablement aussi macédonienne. Et l'espoir, ici, en Béotie, d'un nouveau Leuctres. Mais plutôt que de voir dans le récit les traces directes d'une propagande anti-romaine, mithridatique, regardons la suite.

L'autre thème dominant de l'histoire des Leuctrides est celui du déni de justice. Damon s'est fait justice lui-même. Là aussi l'affaire n'est pas simple. Le crime puni n'avait pas été commis, et Damon n'étant pas non plus Mattathias sur la place de $\operatorname{Modin}^{61}$, le meurtre au sacrifice dans le domaine grec n'a pas que des connotations positives, même s'il se justifie, au plan tactique, du fait que les victimes y sont «nues », sans armes. C'est plutôt une tradition tyrannique $^{62}$. La réponse de la cité n'est pas non plus sans ambiguités : «Face au

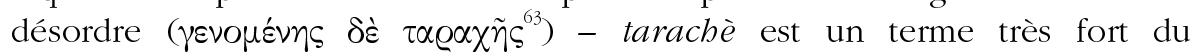
vocabulaire de la stasis -, le conseil des Chéronéens se réunit et condamna à mort les meurtriers». Certes, mais Plutarque d'ajouter aussitôt : «C'était, pour la cité, le moyen de se disculper aux yeux des Romains ». Ruse, secrète complicité avec les jeunes, s'est-on demandé, ou désir compréhensible d'éviter les représailles romaines, ou lâcheté, opportunisme, collaboration ${ }^{64}$ ? La réaction de Damon ne fit pas, pour sa part, dans la nuance : «Un soir (ou le même soir, si l'on tient à dramatiser encore plus ${ }^{65}$ ), comme les magistrats prenaient leur repas en commun, selon la coutume, Damon et ses compagnons firent irruption dans l'archeion, les égorgèrent et s'enfuirent à nouveau de la

\footnotetext{
${ }^{60}$ Plutarque connaît le danger pour un homme politique grec sous l'Empire d'agiter devant les masses les souvenirs de Marathon et autres exploits contre les Perses (Préceptes politiques, 814 a-c); cf. Franco, l.c. (n. 5), p. 197. Damon prolongeant la lutte de ses ancêtres contre les Barbares, Perses puis Galates, et éventuel libérateur et refondateur de la Béotie : MACKAY, l.c. (n. 5), p. 98-99. Dans la bouche de Plutarque, dans le contexte de la guerre mithridatique en Béotie, pour les deux batailles de Chéronée et d'Orchomène, le qualificatif de "Barbares » concerne exclusivement les troupes pontiques (Sylla, 15, 3; 16, 5; 18, 1, et 7; 19, 7 et 10; 21, 8)

1Maccabées 2, 15-28 (avec, à la fin, en 28: "Lui-même et ses fils s'enfuirent dans les montagnes, abandonnant tout ce qu'ils possédaient dans la ville »).

Cf. J. LABARBE, «Un putsch dans la Grèce antique : Polycrate et ses frères à la conquête du pouvoir », AncSoc 5 (1974), p. 21-41.

63 Cimon, 1, 5. Tarachè est un terme codé pour dire le trouble politique, l'état de stasis. C'est l'exact contraire d'bèsychia, la «tranquillité ». Voir, par exemple, pour un contexte proche (Thèbes en 171), Polybe, XXVII, 1, 7-8 et XXVII, 5, 8 .

64 Désir d'éviter les représailles romaines : MA, l.c. (n. 1), p. 62-63, d'après KeAvENEY, o.c. (n. 10), p. 18 et n. 10; décision des pro-Romains au pouvoir : MACKAY, l.c. (n. 5), p. 92; attitude ambiguë: Franco, l.c. (n. 5), p. 200-201; trahison aux yeux des jeunes: ThORNTON, o.c. (n. 5), p. 224-225. Sur le danger de s'opposer aux Romains, cf. infra, n. 88.

MACKAY, l.c. (n. 5), p. 92, 101.
} 
ville »". Meurtre au prytanée. La jeunesse de la cité ne revient pas du séjour sur les marges pour s'intégrer à la polis, mais égorge ses anciens ${ }^{67}$.

C'est ici qu'intervient Lucullus : "Or il se trouva que ces jours-là, Lucius Lucullus passait par Chéronée avec des troupes pour une certaine mission. Il interrompit sa marche, enquêta sur les événements qui venaient de se passer, et découvrit que la cité n'était coupable de rien : au contraire, elle aussi, avait été victime. Il retira les soldats et les emmena avec lui »". Suite à l'enquête judiciaire de Lucullus, la cité obtient donc un répit. L’une des causes de trouble est retirée, la garnison; l'autre demeure, Damon. À noter que le texte peut paraitre ambigu, et a fait l'objet d'interprétations opposées : Lucullus a reconnu que la cité n'était pas responsable du meurtre des Romains et avait été lésée, à son tour et comme eux, par le meurtre des magistrats ${ }^{69}$, ou Lucullus a reconnu que la garnison et son chef, par leur conduite vis-à-vis de la population et donc du jeune Damon, étaient en grande partie responsables des troubles, et que la cité avait été plutôt victime, comme Damon également ${ }^{70}$. En ce sens iraient les épanchements de remerciements de la cité de Chéronée, dans un décret inscrit à Delphes, pour une garnison de cavaliers thraces et son chef Amatokos, laissés cette fois par Sylla l'hiver suivant, et il est noté, avec insistance, de quelle bonne manière, eux, s'étaient conduits envers la population ${ }^{71}$.

Restait le problème Damon : «Cependant Damon ravageait le territoire (la chôra) par ses brigandages (lèisteiai) et ses incursions et harcelait la cité : les

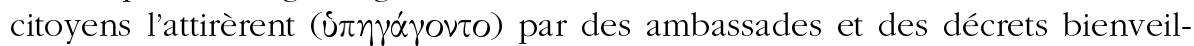
lants et, quand il fut rentré, ils le nommèrent gymnasiarque; après quoi, un jour qu'il s'enduisait d'huile à l'étuve, ils le tuèrent» ${ }^{72}$. Tout ce passage a été jugé le plus problématique, dans la succession ramassée de ses quelques lignes. Et il est celui qui permet le mieux de saisir le jeu moderne des interprétations et des méthodes employées, et leurs enjeux. On a refusé la version de Plutarque, caractérisée par sa rapidité (sauf Mackay qui s'en réclame pour sa chronologie courte ${ }^{73}$ ) et pour l'interprétation qu'il donne de

66 Cimon, 1, 5.

${ }^{67}$ MA, l.c. (n. 1), p. 53. Dans le cadre de la tradition sur Leuctres, on rappellera l'exploit, qui donne le branle à toute la saga de la libération, de Pélopidas et de ses onze compagnons revenus secrètement d'Athènes : le soir, déguisés en femmes, ils tuent les magistrats, les «tyrans », prolacédémoniens de Thèbes, au symposion, alors qu'ils faisaient la fête (Xénophon, Helléniques $\mathrm{V}$, 4; Plutarque, Pélopidas, 6-13; Le démon de Socrate, 587b-588a, 594b-598f). Il y a d'ailleurs à cette occasion un autre jeune garçon qui mériterait d'être rapproché de Damon (Pélopidas, 9, 10-12; Le démon de Socrate, 595b). Toutefois, dans la Vie de Cimon, le deipnon des magistrats de Chéronée, dans le cadre de leurs devoirs civiques, n'est pas signe de débauche, mais assurément une affaire sérieuse, pleine de gravité.

68 Plutarque, Cimon, 1,6

69 MACKAY, l.c. (n. 5), p. 92; THORNTON, o.c. (n. 5), p. 226.

70 Franco, l.c. (n. 5), p. 204-205, 208.

71 Holleaux, o.c. (n. 5), I, p. 144 (1. 5-14 du décret) et 152.

72 Cimon, 1, 7.

73 MACKAY, l.c. (n. 5), p. 103 
la conduite de la cité : une conduite rusée visant à tromper Damon, pour mieux s'en débarrasser. Au contraire, la conduite de la cité a été jugée incohérente, contradictoire, «quasi schizophrénique $»^{74}$. Pour lui redonner sa cohérence, on l'a attribuée à la succession rapide au pouvoir de factions radicalement opposées, pro-romaines et pro-pontiques (Mackay), ou dans une temporalité plus lente (Thornton, Franco), séparées par des écarts plus discrets et engagées entre elles dans un jeu plus subtil ${ }^{75}$. Ces interprétations ont au moins l'avantage de nous faire mieux saisir a contrario ce qu'est celle de Plutarque. La cité (polis, politai) est prise comme un sujet unique, auteur de toutes les actions ${ }^{76}$. Et Plutarque dévoile effectivement son parti-pris, un peu plus loin, quand il va feindre de s'étonner que les Orchoméniens aient intenté un procès en justice, une dikè phonou, à la cité de Chéronée, comme si elle était une seule personne (

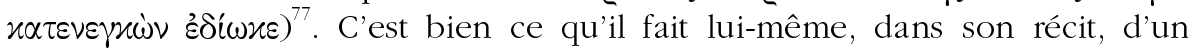
bout à l'autre, de la traiter comme un personnage unique. L'apparente contradiction de la conduite de la cité est ici unifiée sous l'espèce de la ruse. Le verbe íráyelv relève bien du vocabulaire de la ruse et de l'embuscade ${ }^{78}$, et Damon se retrouve assassiné gumnos, comme il avait assassiné le Romain. En un sens, c'est bien la cité qui retourne à des conduites de jeunes, y régresse, serait-on presque tenté de dire ${ }^{79}$. Enfin, l'autre grande caractéristique du récit, comme l'a remarqué à juste titre $\mathrm{C}$. Franco, est sa quasi totale décontextualisa$\operatorname{tion}^{80}$. Presque rien ailleurs dans la Vie de Lucullus ne permet de comprendre ce que ce dernier vient faire par là, "pour une certaine mission », et il nous faut pour cela faire le rapprochement avec un passage de la Vie de Sylla ${ }^{81}$. Aucune référence dans notre texte à Mithridate, Archélaos, Braetius Sura, Sylla, aux batailles de Chéronée et d'Orchomène, tout ce sur quoi il nous donne, au contraire, les détails les plus précis dans l'autre Vie, celle de Sylla. Certes une présence militaire : cette cohorte, les troupes de Lucullus, mais on ne se douterait pas que des dizaines de milliers d'hommes s'égorgent en arrière-plan. On peut comprendre que Plutarque ait des raisons de passer complètement sous silence l'existence d'une faction active pro-pontique à Chéronée, mais il ne dit rien ici non plus de ce dont il parle explicitement ailleurs, des partisans des Romains, des troupes chéronéennes sans doute présentes au siège d'Athènes avec Sylla et que nous retrouvons à la bataille

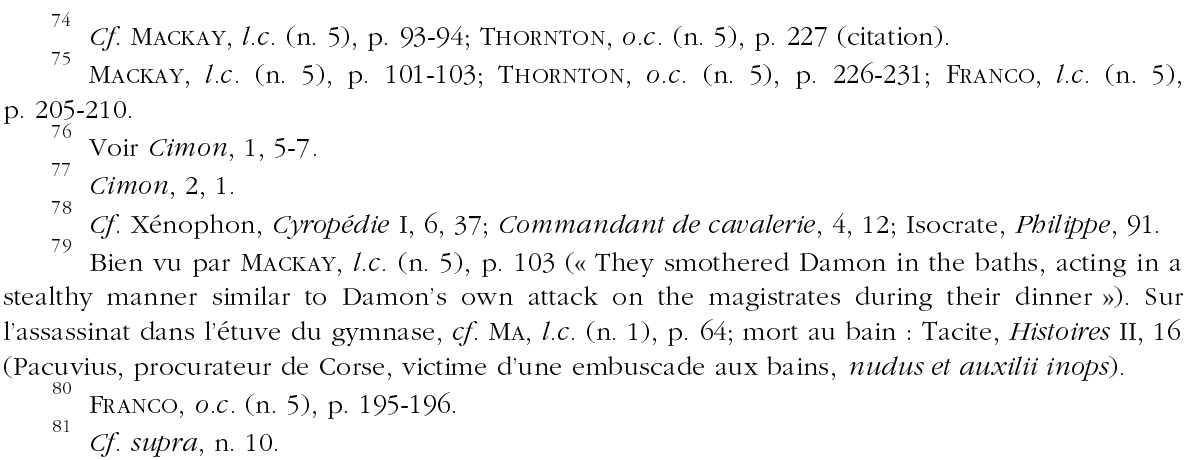


de Chéronée, d'Homoloïchos et d'Anaxidamos (le premier était peut-être son ancêtre), qui obtinrent de protéger la cité des troupes d'Archélaos, puis chassèrent les Pontiques de la position stratégique de la colline de Thourion dominant la plaine au sud. À eux Sylla, pour cet exploit décisif, permit d'élever le trophée que l'on a récemment retrouvé et où ils gravèrent maladroitement leurs noms ${ }^{82}$. D'un côté, dans la Vie de Sylla, le maximum de précision et de détails, avec les Mémoires du général lus sur le terrain. Ici, le dépouillement le plus total, un théâtre au décor le plus simplifié possible pour laisser sur la scène, face à face deux à deux, les trois personnages principaux, Damon, la cité, Lucullus; l'officier romain, qui n'a même pas droit à un nom, et les lamentables Orchoméniens n'étant que des comparses. Damon et Lucullus ne se trouvant jamais ensemble, nous y gagnons de comprendre que, selon les règles du théâtre antique, le véritable protagoniste est la cité.

Damon liquidé, on pourrait croire que les malheurs de la cité sont terminés. Au contraire, c'est maintenant qu'elle va tomber dans le pire danger. Au reste, pour que l'histoire se termine en ce point, en une sorte de résolution et d'apaisement, il aurait fallu au moins que justice soit rendue à Damon, avec un nouveau procès pour meurtre. Mais voit-on la cité se juger elle-même de son crime? Le procès va bien venir, mais d'ailleurs, et elle est accusée du meurtre, non pas de Damon, mais de celui des Romains tués par Damon, comme complice de celui qu'elle avait récompensé ensuite de la charge de gymnasiarque : «Les habitants d'Orchomène, qui étaient voisins et rivaux des Chéronéens, achetèrent les services d'un délateur romain qui intenta un procès à la cité, comme si elle était un simple particulier, et la poursuivit en justice pour le meurtre des hommes que Damon avait assassinés. L'affaire fut portée devant le proconsul (stratègos) de Macédoine (les Romains n'envoyaient pas encore de gouverneurs [stratègoi] en Grèce). Ceux qui parlèrent pour la cité invoquèrent le témoignage de Lucullus, auquel le proconsul écrivit et qui confirma la vérité. La cité fut donc acquittée après avoir couru les dangers les plus grands ${ }^{83}$. On a pensé, d'une part, que les Orchoméniens attaquaient pour régler des problèmes frontaliers, sur un fond de vieilles haines $^{84}$; d'autre part, on s'est demandé ce que risquaient vraiment les Chéronéens : la perte des privilèges éventuels obtenus de Sylla ${ }^{85}$, la condamnation de quelques notables pour l'exemple, ou une épuration plus sauvage, disparaître en tant qu'entité politique et être annexés par Orchomène ${ }^{86}$. En

82 Plutarque, Sylla, 16, 14-15; 17, 6-12; 18, 1-3; 19, 10; Pausanias, IX, 40, 7. Pour le trophée, cf. J. Camp, M. Ierardi, J. Mcinerney, K. Morgan, G. Umholtz, «A Trophy from the Battle of Chaironeia of 86 B.C. », AJA 96 (1992), p. 443-455 (p. 447 n. 9, pour Homoloïchos, éventuel arrière-arrière-grand-père de Plutarque), avec les rectifications de C.S. MACKAY, «Sulla and the Monuments: Studies in his Public Personna, II. 'Discovery' of a Monument of Sulla's at Chaeronea », Historia 49 (2000), p. 168-177.

83 Cimon, 2, 1.

${ }_{85}$ MA, l.c. (n. 1), p. 64-65; Franco, l.c. (n. 5), p. 210-212.

85 KALlET-MarX, o.c. (n. 5), p. 281, qui pense qu'elle avait dû obtenir la « liberté » de Sylla, tout comme Élatée voisine (Pausanias, X, 34, 2).

${ }^{86}$ MA, l.c. (n. 1), p. 67. 
tout cas, il est clair que Plutarque n'a pas choisi la version minimale,

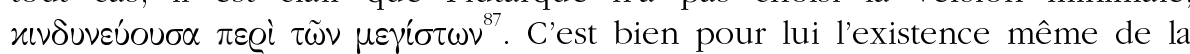
cité qui est en question. Dans le contexte des «traditions » et comportements locaux - car, après tout, ce ne sont pas les Romains qui engagent ce procès -, on peut songer, en remontant au-delà du sort subi de la part de ceux-ci par Haliarte, ou Coronée ou Thisbé dans la III $^{\mathrm{e}}$ guerre de Macédoine ${ }^{88}$, à des épisodes antérieurs, à Platées, Thespies et Orchomène, détruites par Thèbes, ou, réciproquement, à Thèbes détruite par Alexandre aux applaudissements enthousiastes des autres Béotiens ${ }^{89}$. Autrefois, ces choses-là se réglaient au moins par les armes; maintenant on utilise un délateur et un tribunal romain. On est bien entré dans une autre époque.

Si je rassemble les fils de ma démonstration, il me semble que l'illustration de ces tristes temps n'est pas seulement Damon qui manifeste son caractère ambigu et contradictoire, à la fois fier et trop rude, à travers d'abord le meurtre du Romain pour venger préventivement son honneur, puis celui des magistrats, ce qui peut être considéré encore de manière positive, comme une imitation de l'exploit de Pélopidas mettant fin au règne des tyrans de Thèbes asservis à la domination spartiate, ou au contraire comme le pire forfait d'une stasis, avant de sombrer dans le brigandage (lèisteia), pour finalement se laisser prendre aux sirènes de la normalité, de l'illusion d'une carrière honorable, comme s'il avait fait jusque-là un parcours sans faute. C'est aussi la conduite de la cité qui, parallèlement à celle de Damon, peut paraître sans doute pas toujours injustifiée, elle non plus, mais tout aussi ambiguë et contradictoire : incapable de défendre ses jeunes, les condamnant à mort, les acculant à la rébellion, puis, pour s'en débarrasser, s'abaissant à des conduites rusées dignes d'un crypte, amnistiant et récompensant même celui qu'elle a condamné à mort, pour ensuite le liquider subrepticement. Et en même temps, elle aussi victime, et cherchant légitimement à préserver son existence. C'est le cas de dire avec Plutarque qu' "elle était vraiment mal en point ", $\lambda u \pi \varrho \grave{\alpha}$

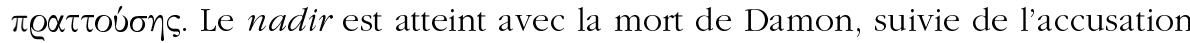

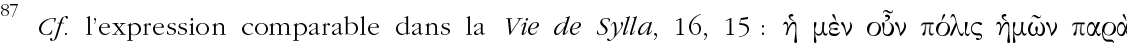

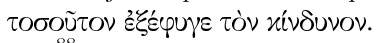

${ }^{88}$ En 196 av. J.-C., pour le meurtre de soldats romains, Flamininus ordonne le siège d'Acraiphiai; les coupables sont livrés, avec, en plus, une forte amende; le territoire de Coronée aussi est ravagé (Tite-Live, XXXIII, 29, 6-12). En 171, Haliarte, qui avait pris le parti de Persée, est prise d'assaut par les Romains et les Thébains, partie des défenseurs massacrés, 2500 survivants réduits en esclavage, la cité rasée et le territoire donné aux Athéniens. Coronée qui l'avait secourue est mise à sac, la population vendue, mais un décret du Sénat annule la décision (TiteLive, XLIII, 3, 7 et 4, 11, et l'épitomé; J. FOsSEY, "The Cities of the Kopaïs in the Roman period », $A N R W$ II, 7.1 [1979] p. 555-556, 567). Après Pydna, à Thisbé, les familles des opposants aux Romains sont vendues (Tite-Live, XLII, 63, 12; MACKAY, l.c. [n. 5], p. 99 n. 30). Il s'agit de faits directement en relation avec la guerre; mais les gens d'Orchomène devaient chercher à montrer que Chéronée avait été antiromaine. Voir aussi J.-L. FERRARY, «Le statut des cités libres dans l'Empire romain à la lumière des inscriptions de Claros », CRAI (1991), p. 569-570.

${ }^{89}$ Platées, détruite en 427 et 373, privée d'existence de 427 à 386 et de 373 à 335; Thespies, annexée en 373; Orchomène, détruite en 364, rétablie seulement après la victoire de Philippe à Chéronée; Thèbes, sans existence de 335 à 316; voir EllingER, o.c. (n. 16), p. 301, 325.
} 
d'Orchomène. Mais c'est au moment où la cité frôle la mort qu'elle est sauvée. En un sens, le vieux schéma initiatique qui a échoué pour Damon, réussit pour la cité, qui émerge à une nouvelle existence, non pas adulte, mais d'une cité dans et de l'empire romain. Bien sûr, il fallait que Damon disparaisse pour qu'apparaisse le nouveau sauveur, Lucullus. Ceci permet aussi de répondre à la question posée précédemment: Rome serait-elle la nouvelle citétyran? Si l'on compare l'histoire avec celle des Leuctrides, la différence est claire : quelles que soient les similitudes apparentes, au déni de justice des Lacédémoniens - au refus d'écouter des cinq éphores, puis des deux rois, puis du moindre citoyen - répond l'accumulation des procédures judiciaires, l'enquête impartiale de Lucullus, puis la possiblité de se défendre auprès du proconsul, la consultation de Lucullus par celui-ci, et son deuxième témoignage qui n'est pas une faveur trompeuse, mais "atteste la vérité »,

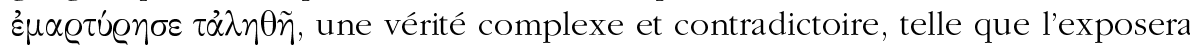
Plutarque à propos de Lucullus lui-même, sans cacher ses moins beaux côtés, car «il n'aurait pas accepté d'être récompensé de son témoignage véridique par un récit mensonger et fictif de ce qu'il fut »".

Tout est-il donc bien qui finit bien dans le meilleur des mondes romains possibles? Il reste tout de même une ombre, c'est le cas de le dire. Dans l'étuve du gymnase, là où Damon avait été tué, "pendant longtemps, on vit des fantômes et l'on y entendit des sanglots, à ce que disent nos pères ${ }^{91}$ " - la parole respectable de la génération précédente est garante de cette affirmation. La cité avait érigé une statue de son sauveur sur l'agora à côté de celle de Dionysos, laissant sans doute entendre qu'il était lui aussi le Nouveau Dionysos, mais elle avait visiblement oublié de juger ou de venger le meurtre de Damon. Un fantôme au gymnase, mauvaise fréquentation à tout point de vue pour les jeunes ${ }^{92}:$ "On fit donc murer l'étuve». La cité s'obstinait dans la même ligne. En vain : «De nos jours encore, ceux qui habitent au voisinage croient que ce lieu est hanté par des apparitions et des voix troublantes»,

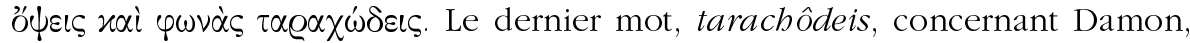
est le même que le premier qui résultait de son acte, tarachè, le désordre dans la cité $e^{-93}$.

Mais ce n'est pas tout. Il y a un autre reste, cette fois hors de Chéronée : «Il subsiste aujourd'hui, surtout à Stiris en Phocide, quelques descendants de la famille de Damon, qui parlent le dialecte éolien, et on les appelle Asbolômenoi ("Couverts de suie »), car Damon, avant le meurtre, s'était frotté de suie ». Stiris, à 120 stades au sud-ouest de Chéronée, par la montagne, était probablement la base arrière de Damon et de sa bande. Et quand ils furent définitivement exilés, ils s'y étaient réfugiés, y obtenant sans doute la

90 Cimon, 2, 3.

91 Cimon, 1, 8.

92 Mauvaise fréquentation politique sûrement, si l'on en croit THORNTON, o.c. (n. 5), p. 233, 247. Sur les autres dangers présentés par les fantômes des bains (dans un contexte chrétien), C. BONNER, «Demons of the Bath », in Studies presented to F.L. Griffith, Oxford, 1932, p. 203-208.

93 Cimon, 1, 8; cf. 1, 5. 
politeia $^{94}$. Mais ils mettaient encore leur point d'honneur, dans la Phocide dorienne, à garder leur dialecte béotien, et peut-être se jugeaient-ils les seuls véritables Chéronéens ${ }^{95}$. Mais surtout leur nom doit nous interpeller. Les Asbolômenoi: il faut le lire en regard de dénominations du même type, d'abord les aleiphomenoi, l'association cultuelle des jeunes du gymnase des cités hellénistiques ${ }^{96}$, ou ces Psapharoi, les "Poudreux», que l'on a retouvés récemment à Laodicée du Lycos, honorant Zeus et Hermès ${ }^{97}$. Dans la voisine même de Chéronée, Orchomène, nous apprend Plutarque, dans les Questions grecques, il existait un genos des Psoloeis, les «Couverts de cendres, de suie noire », voulant dire cette fois les «Endeuillés», ceux qui ont maculé leurs vêtements en signe de deuil : il s'agissait des descendants des époux des Minyades rendues folles par Dionysos pour n'avoir pas voulu honorer son culte et qui avaient déchiré, comme l'on sait, leurs propres enfants. Les descendants des Minyades elles-mêmes constituaient un genos parallèle, dont les femmes étaient appelées Oleiai, les «Meurtrières ». À la fête des Agrionies, elles fuyaient devant le prêtre de Dionysos, choisi dans l'autre lignée. Celui-ci, armé d'une épée, avait le redoutable privilège de mettre à mort celle qu'il rattrapait $^{98}$. Les Asbolômenoi ne sont donc pas simplement des aleiphomenoi en négatif, mais forment un genos dont le nom honore ce qui pour eux n'était certainement pas le crime, mais l'exploit fondateur de leur ancêtre Damon. Rapprochons les éléments disjoints par l'espace: d'un côté, en Béotie, à Chéronée, un fantôme emmuré au gymnase; de l'autre, en Phocide, à Stiris, un genos et le culte héroïque de son fondateur. Si l'on superpose les deux, on obtient la figure en creux d'une réalité typique de cette basse époque hellénistique, ou si l'on préfère du début de l'époque romaine dans le monde grec, de l'évergète, sauveur, nouveau fondateur, enterré au gymnase, cette deuxième agora dont parlait Louis Robert" ${ }^{99}$ Face à Lucullus et à sa statue de

\footnotetext{
94 En tant que valeureux patriotes béotiens ou pour leurs lucratifs brigandages ? Damon avait eu aussi des descendants, faut-il croire, de la sœur d'un de ses compagnons ou de quelque jeune Phocidienne; voir les réflexions de $\mathrm{Ph}$. GAUTHIER, Les cités grecques et leurs bienfaiteurs, Athènes/Paris, 1985 (BCH, Suppl. 12), p. 165-166, sur la politeia potentielle accordée par la cité de Lilaia de Phocide, vers 211-208, à des mercenaires du roi de Pergame laissés par celui-ci pour la protéger contre Philippe $\mathrm{V}$, et sur les relations avec la population.

95 Cf. MACKAY, l.c. (n. 5), p. 103 n. 43 : «pathetically clinging to their Boeotian dialect. »

Cf. IG XII, Suppl. 122 : Éresos, où le gymnasiarque à la fois mène les jeunes autour des zones frontières et préside au banquet des aleiphomenoi à l'occasion des Herméia (cité par MA, l.c. [n. 1], p. 54).

Th. CORSTEN (éd.), Die Inschriften von Laodikeia am Lykos I, Bonn, 1997 (Inschriften griechischer Städte aus Kleinasien, 49), $\mathrm{n}^{\circ}$ 63; cf. C. BRIXHE, «Bulletin épigraphique », REG 111 (1998), n 433. Les athlètes grecs s'enduisaient à la fois d'huile (aleiphomenoi) et de poudre, de poussière. Hermès est le dieu du gymnase.

98 Questions grecques, 38, 299e-f, avec l'incident désastreux du prêtre qui osa exercer son droit.

99 Voir ainsi les exemples de Callicratès fils de Pythôdoros à Aphrodisias (J. REynOLDS, Aphrodisias and Rome, Londres, 1982 [JRS Monographs, 1], p. 150-156; C. VIAL, Les Grecs de la paix d'Apamée à la bataille d'Actium, Paris, 1995, p. 207-208), d'Artémidoros, fils de Gaius Julius Théopompe le mythographe, à Cnide (W. BlüMEL, Die Inschriften von Knidos. I, Bonn [IK 41],
} 
sauveur sur l'agora, la figure disloquée de Damon en est bien en un sens le double, l'ombre portée, une virtualité qui n'a pu se réaliser, en lui cédant la place, que dans la figure opposée du Romain.

J'ai rétabli dans mon exposé l'ordre chronologique. Plutarque, au contraire, a terminé avec Lucullus, voulant privilégier le côté positif des choses. Mais nous a-t-il laissé entendre, par anticipation, tout autant que les bienfaits de Lucullus, le fantôme est toujours là présent, même sous une forme atténuée. Mais laisser indéfiniment subsister un tel élément de désordre, la cité grecque " normale », celle des récits de la tradition sur le temps d'autrefois, l'aurait-elle permis? Murer le fantôme était-il vraiment la procédure adéquate? Un phénomène de ce type entre dans la catégorie des conséquences de l'agos, comme un loimos dont il peut être d'ailleurs la manifestation. La cité consulte alors généralement l'oracle de Delphes, qui prescrit le type de purification nécessaire. Quel est-il pour un fantôme ? Sans aller chercher aussi loin que les kolossoi de la Grande loi sur les Suppliants de Cyrène, la voisine Orchomène nous donne une fois de plus une idée de la solution. Comme le raconte Pausanias, le fantôme d'Actéon ravageait la chôra en roulant une énorme pierre. L'oracle de Delphes consulté prescrivit de rassembler ce qui pouvait subsister de son corps dévoré par les chiens, de lui donner un tombeau et d'ériger une statue de bronze à l'image du fantôme que l'on attacherait par des chaînes à la pierre en question, et de lui sacrifier désormais comme à un héros $^{100}$. De même, à Siris, on avait conjuré les mânes de cinquante jeunes gens massacrés à l'autel d'Athéna en leur érigeant des statues ${ }^{101}$. Pour apaiser le fantôme du régent Pausanias de Sparte qui hantait le sanctuaire d'Athéna Chalkioikos, on avait élevé deux statues de bronze près de l'autel ${ }^{102}$. Cela, je pense, celui qui n'était pas seulement le principal notable de Chéronée, mais le prêtre à vie d'Apollon à Delphes, ne pouvait pas l'ignorer ${ }^{103}$. Les Chéro-

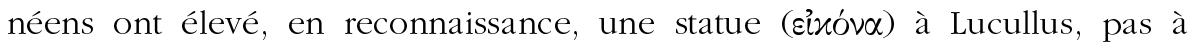
Damon. Plutarque, à son tour, qui considère, bien que de nombreuses générations le séparent de Lucullus, que cette dette de reconnaissance le concerne encore, lui et ses concitoyens, « les vivants d'aujourd'hui », pour l'acquitter, a décidé de lui «élever une statue ( $\operatorname{ixóv\alpha )~encore~plus~belle~que~celle~qui~}$

1992, n 59, 1. 9-11), de L. Vaccius Labeo à Kymè d'Éolide (H. Engelmann, Die Inschriften von Kyme, Bonn, 1976 [IK 5], n 19, 1. 7, 10-11, 49-52); cf. L. ROBERT, « Inscriptions d'Aphrodisias », AC 35 (1966), p. 420-423 (= OMS VI [1989], p. 44-47); Ph. GAUTHIER, o.c. (n. 94), p. 61-66.

100 Pausanias, IX, 38, 5.

101 Justin, XX, 2.

102 Aristodèmos, FGrHist $104 \rrbracket 8$, 5; Lettres de Thémistocle, 4, 15-18 (éd. G. CORTASSA et E. C. Gastaldi, Le Lettere di Temistocle, Padoue, 1990, I, p. 56 et 109) : Thémistocle menace de devenir pour la cité d'Athènes qui se souillerait de sa mort un fantôme vengeur, palamnaios, alitèrios, ou alastôr, pire que Pausanias, plus terrible encore que la malédiction de Cylon, et que ne pourraient même pas conjurer des statues de bronze.

103 Plutarque et la conjuration du fantôme du régent Pausanias : Sur les délais de la justice divine, 560e; fr. 126 Sandbach, (Moralia, vol. XV, coll. Loeb, p. 240-243); réfléchissant sur ce type d'êtres surnaturels : Sur la disparition des oracles, 15, 418b-c. Pour ces rituels, cf. D. OGDEN, Greek and Roman Necromancy, Princeton, 2001, p. 100-107. 
reproduit seulement l'apparence extérieure du corps et du visage», celle qui "met en lumière le caractère et le comportement", une statue de mots qui est la biographie qu'il va écrire de lui ${ }^{104}$. Or pour expliquer pourquoi il était nécessaire de rendre hommage à Lucullus, il fallait bien parler de Damon. De fait, la caractéristique de ce curieux prologue que l'on a qualifié de digression en rapport avec le sujet ${ }^{105}$, est bien d'être, malgré sa brièveté, une biographie complète de Damon, avec les origines et la situation familiales, la définition du caractère, les actions, la mort, et jusqu'à l'évocation des descendants et du rapport au temps présent par quoi se clôt souvent une biographie de Plutarque $^{106}$. La paire des Vies ici en contient en fait trois, celle de Cimon, celle de Lucullus, plus celle de Damon, une biographie miniature, une petite statue de mots. Je ne dirai sans doute pas qu'on a là un kolossos, une figurine de substitution, et dans ces pages un rituel de conjuration d'un fantôme. De toute façon, elles entretiendraient avec lui la même relation métaphorique que la biographie de Lucullus avec la statue de l'agora. Mais peut-être peut-on aller un peu plus loin. Entre Lucullus et Chéronée s'est probablement établi un rapport de l'ordre du patronat, qui, deux cents ans après, n'est plus que symbolique $^{107}$ - et cela, même si Plutarque ne le dit pas en des termes techniques, comme les néologismes patrôn et patrôneuein forgés par les cités des II $^{\mathrm{e}}$ et $\mathrm{I}^{\text {er }}$ siècles pour exprimer la nouvelle réalité qui dépassait l'ancien évergétisme ${ }^{108}$. Or si Plutarque, au nom des siens, se sent une dette de reconnaissance envers le patron de sa cité, qu'en est-il vis-à-vis de Damon ? Plutarque se targuait de descendre, sinon tout à fait directement, du moins le clan familial au sens large dont il était le plus éminent représentant, non seulement de Daïphantos d'Hyampolis, le libérateur de la Phocide, mais aussi d'Opheltas, le roi re-fondateur de la Béotie ${ }^{109}$. Comment penser la relation de

104 Cimon, 2, 2. Le même type de considérations, dans Périclès, 2, s'engage également à propos de statues. L'habituelle traduction du deuxième sixóv $\alpha$ par "image " ou "portrait " anticipe un peu trop vite le glissement subséquent vers la comparaison entre écriture et peinture. Autre statue de mots dans J. Scheid, J. Svenbro, «Le mythe de Vertumne », Europe, 904-905 (août-septembre 2004) : Mythe et Mythologie dans l'Antiquité gréco-romaine, p. 174-189.

105 Ph. A. STADTER, «The Proems of Plutarch's Lives », ICS 13 (1988), p. 290-291.

106 Typique d'une fin de Vie; cf. Thémistocle, 32, 6; Antoine, 87, 9; Aristide, 27, 7; Aratos, 54, 8; Pelling, o.c. (n. 43), p. 367 et 369.

107 FRANCO, l.c. (n. 5), p. 212

108 Cf. J.-L. FERRARY, "The Hellenistic World and Roman Political Patronage », in P. Cartledge, P. Garnsey, E. Gruen (éds.), Hellenistic Constructs. Essays in Culture, History, and Historiography, Berkeley, 1997, p. 105-119. Pour Lucullus lui-même, qualifié de "patron », en même temps que de sauveur, bienfaiteur, fondateur, ou protecteur, à Éphèse, Thyatire, Synnada, Délos et autres cités, voir maintenant J.-L. FERRARY, "Les inscriptions du sanctuaire de Claros en l'honneur de Romains », BCH 124 (2000) p. 338-340, en particulier n. 15 et 20, où l'ensemble des références sont réunies. Plutarque reste très sobre en disant seulement que Lucullus a sauvé Chéronée ( $\sigma \omega \theta \varepsilon \dot{v} \tau \varepsilon \subseteq$ ), tandis que le vocabulaire codé des décrets réapparaît dans Pélopidas, 12, 7 ,

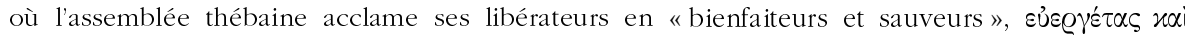
$\sigma \omega \tau \tilde{\eta} \varrho \alpha \varsigma$.

109 Des délais de la justice divine, 558a-b. Cf. B. EINARSON, "Plutarch's Ancestry », CPh 47 (1952), p. 99; CPh 50 (1955), p. 253-255; K. ZIEGLER, "Plutarchs Ahnen», Hermes 82 (1954), p. 499-501; C.P. Jones, Plutarch and Rome, Oxford, 1972, p. 8, 40. 
l'Opheltiade et du dernier des Péripoltides, comme des deux genè ? C'est celle qui découle de la relation du roi et du devin. Au devin est confiée la tâche de résoudre des crises qui dépassent l'intelligence purement politique ou tactique, mais la supériorité doit rester en tout état de cause du côté du chef. La perpétuation d'un tel rapport est difficile à penser en termes purement grecs, mais peut-être moins en termes romains, et Plutarque, notable grec mais aussi romain ${ }^{110}$, s'est peut-être senti, à distance, quelque devoir non seulement de reconnaissance mais de protection, un sentiment vaguement paternel vis-à-vis du jeune orphelin, et comme une dette aussi à acquitter au nom de la cité $^{111}$. Après une crise inextricable, impossible à démêler par des moyens seulement humains, l'instauration d'un culte hérö̈que permettait autrefois, dans le langage du rite, à la cité d'assumer et d'intégrer ce qui s'était passé dans sa redoutable complexité. En érigeant, discrètement, cette petite statue de mots à Damon, Plutarque a certainement réalisé aussi ce qu'on appellerait aujourd'hui un travail de mémoire - qui n'est jamais à confondre, quoi qu'on veuille trop souvent nous faire croire, avec le travail même de l'historien - aidant sa petite cité à mieux assumer son passé qu'en emmurant le fantôme. Il y a dans ce prologue un double aspect, non seulement de politique extérieure, mais aussi de politique intérieure, contribuant au programme de Plutarque en faveur de l'bomonoia dans la cité, et de présence à l'intérieur de celle-ci, pour éviter que, de petite, elle ne le devienne encore plus $^{112}$.

Après que Plutarque eut écrit ces pages, le fantôme a-t-il cessé de hanter le gymnase ? Je n'oserais l'affirmer, et nous n'aurons pas la cruauté de demander à nos collègues archéologues de nous renseigner là-dessus, si jamais ils venaient à le fouiller. Ils n'auraient peut-être pas plus de succès qu'avec le pneuma delphique. Mais nous sommes tous, chercheur ou simple lecteur, fascinés par cette merveilleuse et emblématique histoire, qui dit le passage et l'intégration de la cité grecque dans l'empire romain, sans masquer sa part d'ombre, et il est loisible à chacun de nous de ressentir par lui-même que Plutarque, par la magie de la littérature, y a fixé quelque chose de l'esprit noble et fier, mais tout de même un peu rude, du malheureux jeune homme de Chéronée.

Pierre ELLINGER

Université Paris 7 - Denis Diderot

UFR Géographie, Histoire et Sciences de la Société

2, place Jussieu - case courrier 7001

F - 75251 PARIS Cedex 05

110 C.P. JONES, o.c. (n. 109), p. 39-47. Plutarque n'était pas seulement citoyen romain, ce qui était dans son milieu banal et à peine digne de mention, mais membre de l'ordre équestre.

111 Patronat et paternité : Plutarque, Fabius Maximus, 13, 6-8

12 Cf. Préceptes politiques, 824c, e; Démosthène, 2, 2; S. SWAIN, Hellenism and Empire. Language, Classicism, and Power in the Greek World, AD 50-250, Oxford, 1996, p. 178-182. 